\title{
Delayed Obstruction of XEN Gel Stent by Cell Debris in Primary Open-angle Glaucoma: A New Insight into the Pathophysiology of Filtration Device Failure
}

\author{
Kevin Gillmann ${ }^{1}$, Giorgio E Bravetti ${ }^{2}$, Kaweh Mansouri ${ }^{3}$
}

\begin{abstract}
Aim: The aim of this report is to explore the risk factors of XEN stent obstruction, suggesting the need for a stricter control of these factors and highlighting areas for further research.

Background: Despite its proven effectiveness and safety profile, XEN gel stents (Allergan PIc, Dublin, Ireland) can become obstructed. The causes and predicting factors for such obstructions still require further research. While hyphema has long been known to be responsible for secondary glaucoma through trabecular obstruction, it has not been associated, to date, with XEN gel stent obstruction.

Case description: We describe the case of a 55-year-old female patient with primary open-angle glaucoma (POAG) who underwent bilateral XEN gel surgery. Her left eye developed a $2 \mathrm{~mm}$ postoperative hyphema, which resolved spontaneously within 8 days. Intraocular pressure (IOP) normalized at $12 \mathrm{~mm} \mathrm{Hg}$ and increased to $50 \mathrm{~mm} \mathrm{Hg}$ after 1 month in an otherwise normal-looking eye. Intraoperative examination revealed a nonfunctioning XEN gel stent, which was replaced and sent for laboratory analysis. Macroscopic examination of the tube confirmed obstruction with cellular debris. Tube replacement restored good filtration.

Conclusion: This case report confirms cellular debris as a potential cause of XEN gel stent occlusion, suggesting that aqueous red blood cells (RBCs) could potentially pose a threat to the microstents' patency even in cases when the bleeding was minimal and self-limited and where the IOP was still controlled at the time of full hyphema resolution. This observation could lead to recommendations for a stricter control of bleeding risk factors prior to microinvasive glaucoma surgery (MIGS), and it raises the question of whether anterior chamber (AC) washout should be advised in postoperative hyphema.

Clinical significance: This case highlights some previously unreported risk factors for XEN stent obstruction and suggests that stricter control of bleeding and monitoring of patients following hyphema could improve surgical outcome.

Keywords: Blockage, Glaucoma, Glaucoma surgery, Hyphema, Microinvasive glaucoma surgery, Obstruction, Red blood cells, XEN.

Journal of Current Glaucoma Practice (2019): 10.5005/jp-journals-10078-1258
\end{abstract}

\section{BACKGROUND}

In recent years, microinvasive glaucoma surgery (MIGS) has emerged as a safer surgical alternative to traditional filtering surgery in mild-to-moderate glaucoma. ${ }^{1}$ Amongst MIGS options, the XEN gel stent (Allergan PIc, Dublin, Ireland) is $6 \mathrm{~mm}$-long tubes, made of crosslinked gelatin. ${ }^{2}$ They were designed to be used $a b$ interno to create a drainage canal through the trabecular meshwork between the anterior chamber (AC) and the subconjunctival space. Its $45 \mu \mathrm{m}$ internal diameter was calculated using Hagen-Poiseuille equation to minimize the risk of hypotony. ${ }^{3}$ However, despite a good safety profile, the very small lumen of the XEN gel stents has a certain propensity to become obstructed. The exact causes and predicting factors for such obstructions still require further research. ${ }^{4,5}$

Hyphema has long been known as a potential cause of secondary glaucoma. ${ }^{6}$ This is assumed to result from clogging of the trabecular meshwork by red blood cells (RBCs) by-products and subsequent fibrotic changes. ${ }^{7}$ Trabecular obstruction secondary to $A C$ hemorrhages has been observed following several routine ophthalmic procedures and microtrauma; ${ }^{8,9}$ however, to date, they have not been associated with XEN gel stent obstruction.

\footnotetext{
${ }^{1-3}$ Department of Ophthalmology, Glaucoma Research Center, Montchoisi Clinic, Swiss Visio, Lausanne, Switzerland
}

Corresponding Author: Kevin Gillmann, Department of Ophthalmology, Glaucoma Research Center, Montchoisi Clinic, Swiss Visio, Lausanne, Switzerland, Phone: +41 216193742, e-mail: kevin. gillmann@wanadoo.fr

How to cite this article: Gillmann K, Bravetti GE, Mansouri K. Delayed Obstruction of XEN Gel Stent by Cell Debris in Primary Open-angle Glaucoma: A New Insight into the Pathophysiology of Filtration Device Failure. J Curr Glaucoma Pract 2019;13(3):113-115.

Source of support: Nil

Conflict of interest: None

\section{Case Description}

In this report, we describe the case of 55-year-old lady of Central African origin with primary open-angle glaucoma (POAG) who underwent bilateral XEN gel surgery.

\section{Initial Presentation}

The patient was initially referred by her general ophthalmologist to a tertiary glaucoma center with intraocular pressures (IOP) of

() The Author(s). 2019 Open Access This article is distributed under the terms of the Creative Commons Attribution 4.0 International License (https://creativecommons. org/licenses/by-nc/4.0/), which permits unrestricted use, distribution, and non-commercial reproduction in any medium, provided you give appropriate credit to the original author(s) and the source, provide a link to the Creative Commons license, and indicate if changes were made. The Creative Commons Public Domain Dedication waiver (http://creativecommons.org/publicdomain/zero/1.0/) applies to the data made available in this article, unless otherwise stated. 
$38 \mathrm{~mm} \mathrm{Hg}$ and $22 \mathrm{~mm} \mathrm{Hg}$ in the right and the left eyes, respectively. She had been diagnosed with POAG several years before and had been managed with two selective laser trabeculoplasties (SLT) and maximal medical therapy including oral acetazolamide (Vifor Pharma, Bern, Switzerland), despite mediocre self-reported compliance. She had a positive family history for open-angle glaucoma in her mother. At the time of presentation, her bestcorrected visual acuity was $10 / 10$ in both eyes on a decimal chart (6/6 Snellen) in slightly myopic eyes ( $-0.75 \mathrm{D}$ and $-1.25 \mathrm{D}$ spherical equivalent in the right and the left eyes, respectively). Slit lamp examination revealed a deep and quiet $A C$ and a clear crystalline lens. Cup/disk ratio was 0.8 in the right eye with an inferior notch and 0.7 in the left eye with a superior notch. Gonioscopy showed bilaterally open angles. Pachymetry was $580 \mu \mathrm{m}$ and $585 \mu \mathrm{m}$ in the right and the left eyes, respectively. Automated visual field examination (Octopus, Haag Streit, Koeniz, Switzerland) showed bilateral nasal quadrantanopsia. Optical coherence tomography imaging (Spectralis OCT, Heidelberg Engineering AG, Germany) of the retinal nerve fiber layer (RNFL) showed generally reduced RNFL thicknesses bilaterally, with complete atrophy of the RNFL inferiorly in the left eye. Brain MRI imaging was unremarkable.

\section{XEN Gel Surgery and Follow-up}

Bilateral mitomycin C-augmented XEN gel stent implantation was organized with a target IOP $\leq 18 \mathrm{~mm} \mathrm{Hg}$. Surgeries were performed following standard protocols described in the literature. No intraoperative complications were noted, and the patient received a standard postoperative treatment of topical combined tobramycin and dexamethasone (Novartis Pharma, Basel, Switzerland) in decreasing regime. The right eye recovered uneventfully, with IOP normalizing between $12 \mathrm{~mm} \mathrm{Hg}$ and $16 \mathrm{~mm} \mathrm{Hg}$ at 3 months, with no additional therapy. The left eye developed a 2-mm hyphema on the first day following surgery, associated with dense RBC in the AC and corneal edema. The initial intraocular pressure was $2 \mathrm{~mm} \mathrm{Hg}$, which gradually improved with scopolamine (OmniVision Pharma, Puchheim, Germany) to $9 \mathrm{~mm} \mathrm{Hg}$ at day 3. After 8 days, the hyphema had completely resolved and IOP was stable at $12 \mathrm{~mm} \mathrm{Hg}$. After 1 month, the patient presented to her postoperative follow-up with an IOP of $50 \mathrm{~mm} \mathrm{Hg}$ in the left eye. On examination, the filtration bleb was shallow but diffuse, the iridocorneal angle was open, the XEN gel stent was well-positioned, and no clear obstruction was visible on its intraocular tip.

\section{XEN Gel Stent Obstruction}

Intraocular pressure was reduced using topical and intravenous medications, and an emergency revision procedure was organized in theater. The absence of filtration through the stent was confirmed intraoperatively and the blocked tube was removed. It was sent for macroscopic analysis to confirm the cause of the obstruction and was replaced by a new XEN gel implant. The patient made good recovery without any postoperative complications, and at 1-month, her IOP was stable at $17 \mathrm{~mm} \mathrm{Hg}$.

The macroscopic examination confirmed obstruction of the XEN gel stent on its AC extremity, with translucent cell fragments (Fig. 1). No fibrin, blood clot, or other type of tissue could be identified within the obstructed tube.

\section{Discussion}

This case report confirms cell fragments as a potential cause of XEN gel stent occlusion. In this specific case, the exact nature of

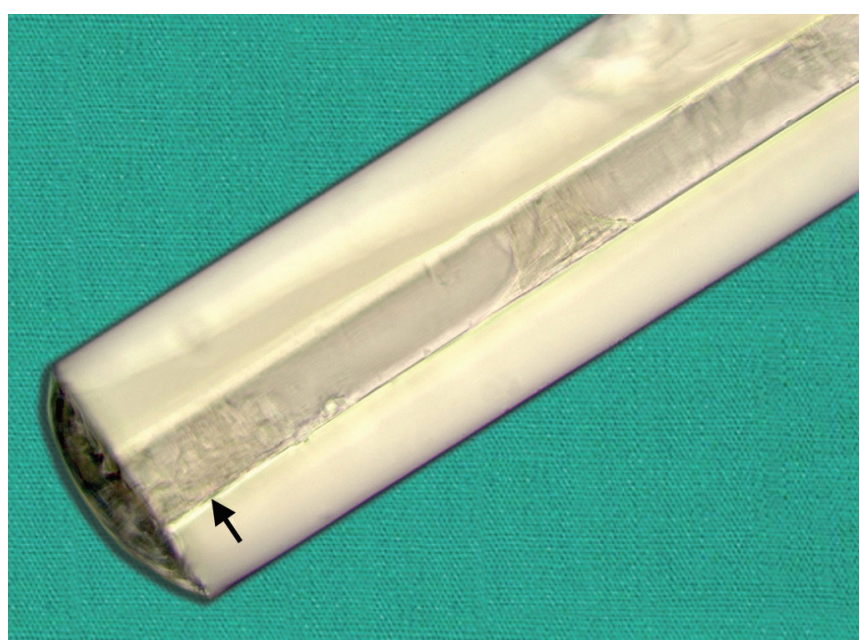

Fig. 1: Macroscopic view of the proximal tip of an obstructed XEN gel stent (Allergan Plc, Dublin, Ireland). Multiple cell fragments can be seen within the $45 \mathrm{~mm}$-wide lumen, with a dense aggregate around its anterior chamber opening (arrow) ( $\times 126$ microscopy, unstained)

the cells debris could not be clearly identified microscopically, and they could originate from any type of cells present within the aqueous. In our opinion, however, several factors draw strong suspicions on the theory of the RBC origin: out of the three XEN gel stent implanted in this patient's eyes, two achieved good and durable IOP control, while one became obstructed within just a few weeks of implantation. The only notable difference between the three procedures was the presence of a hyphema and dense $A C R B C$ following the implantation of the stent that did eventually fail. Interestingly, this suggests that AC RBC can present a potential threat to XEN gel stents' patency, even in cases when the bleeding was minimal and self-limited and where the IOP was still controlled at the time of full hyphema resolution, similarly to what has been observed in sickle cells and ghost cell glaucoma. ${ }^{10-12}$ Clinically, this observation leads to several recommendations. First, unlike fibrin plugs that are usually visible under gonioscopic examination, ${ }^{11}$ cell fragments are translucent and can easily mimic a filtering bleb obstruction that would not, however, respond to needling revision. Second, the success rate of XEN gel surgery could potentially be influenced by higher bleeding risks; thus, patients with known bleeding disorders or anticoagulation therapy should be managed accordingly to minimize the risk of postoperative hyphema. Finally, patients presenting with postoperative hyphema should be carefully monitored for XEN gel stent obstruction, as erythrocyte resorption studies have shown that, while $50-70 \%$ of aqueous RBC are gradually reabsorbed into the blood stream, over a third of the cells gradually break down within the AC over days. ${ }^{13}$ Hence, stent blockage by RBC by-products can develop within 1-9 weeks of an intraocular hemorrhage and can cause a delayed rise in IOP. ${ }^{14}$ More evidence will be needed, however, to determine if early therapeutic actions like an AC washout could reduce the risk of developing subsequent stent obstruction.

\section{Conclusion}

In conclusion, this report confirms that XEN gel stents can become obstructed from cell fragments. It appears that postoperative AC bleed could be a potential risk factor for such type of stent blockage, and every effort should be made to minimize the risk of perioperative bleed. More research 
is needed to determine if and when AC washout should be performed, in the event of an hyphema, to reduce the risk of XEN gel stent obstruction.

\section{Clinical Significance}

This case highlights some previously unreported risk factors for XEN stent obstruction and suggests that stricter control of bleeding and monitoring of patients following hyphema could improve surgical outcome.

\section{References}

1. YookE, Vinod K, Panarelli J. Complications of micro-invasive glaucoma surgery. Curr Opin Ophthalmol 2018;29(2):147-154. DOI: 10.1097/ ICU.0000000000000457.

2. Chaudhary A, Salinas L, Guidotti J, et al. XEN gel implant: a new surgical approach in glaucoma. Expert Rev Med Devices 2017;15(1): 47-59. DOI: 10.1080/17434440.2018.1419060.

3. Mansouri K, Guidotti J, Rao H, et al. Prospective evaluation of standalone XEN gel implant and combined phacoemulsification-XEN gel implant surgery: 1-year results. J Glaucoma 2018;27(2):140-147. DOI: $10.1097 /$ IJG.0000000000000858.

4. Dervenis N, Mikropoulou A, Dervenis P, et al. Dislocation of a previously successful XEN glaucoma implant into the anterior chamber: a case report. BMC Ophthalmol 2017;17(1):148. DOI: 10.1186/ s12886-017-0540-1.

5. Gillmann K, Mansouri K, Bravetti GE, et al. Chronic intraocular inflammation as a risk factor for XEN gel stent occlusion: a case of microscopic examination of a fibrin-obstructed XEN stent. J Glaucoma 2018;27(8):739-741. DOI: 10.1097/JJG.0000000000001002.

6. Sihota R, Kumar S, Gupta V, et al. Early predictors of traumatic glaucoma after closed globe injury. Arch Ophthalmol 2008;126(7):921-926. DOI: 10.1001/archopht.126.7.921.

7. Gragg J, Baker MB. Hyphema. StatPearls. Treasure Island (FL): StatPearls Publishing; 2018

8. Thompson JM, Chang JS, Bermudez-Magner JA, et al. Ghost cell glaucoma following sutureless scleral-fixated posterior chamber intraocular lens placement. Ophthalmic Surg Lasers Imaging Retina 2015;46(1):111-113. DOI: 10.3928/23258160-20150101-22.

9. Elgin U, Sen E, Teke MY, et al. Microtrauma-induced recurrent hyphema and secondary glaucoma associated with chronic acetylsalicylic acid use. Int Ophthalmol 2012;32(1):89-92. DOI: 10.1007/s10792-012-9517-5.

10. Goldberg MF. The diagnosis and treatment of secondary glaucoma after hyphema in sickle cell patients. Am J Ophthalmol 1979;87(1): 43-49. DOI: 10.1016/0002-9394(79)90190-9.

11. Saldanha C. Human erythrocyte acetylcholinesterase in health and disease. Molecules 2017;22(9):E1499. DOI: 10.3390/ molecules22091499.

12. Osman EA, Mousa A, Al-Mansouri SM, et al. Glaucoma after openglobe injury at a tertiary care university hospital: cumulative causes and management. J Glaucoma 2016;25(3):e170-e174. DOI: 10.1097/ IJG.0000000000000162.

13. Hørven I. Erythrocyte resorption from the anterior chamber of the human eye. Acta Ophthalmol (Copenh) 1963;41:402-412. DOI: 10.1111/j.1755-3768.1963.tb03549.x.

14. Campbell DG, Simmons RJ, Grant WM. Ghost cells as a cause of glaucoma. Am J Ophthalmol 1976;81(4):441-450. DOI: 10.1016/00029394(76)90299-3. 